\title{
207 In voice production
}

A The frequency of tone can be altered by adjusting the shape of the free margin of the vocal cords.

B Articulation is performed by the vibrating cords.

C Infraglottic air pressure changes do not alter pitch.

D The neuromuscular theory supposes that vocal cord vibrations are produced by muscle contractions.

E The aerodynamic theory states that vocal cord vibrations are due to infraglottic pressure.

\section{Symptoms and signs of laryngeal disease in the newborn} Include

A Failure to thrive.

B Cough.

C Tracheal plunging.

D Croup.

E Tachycardia.

\section{Congenital laryngeal stridor (laryngomalacia)}

A The laryngeal superstructure is soft and may be oedematous.

B The epiglottis is normal.

C May present as failure to thrive.

D Amputation of the epiglottis is effective treatment.

E The condition usually resolves, without treatment, by age 2 years. 